\title{
The Local Bubble and Interstellar Material Near the Sun
}

\author{
P. C. Frisch ${ }^{1, *}$ \\ ${ }^{1}$ Dept. of Astronomy and Astrophysics, University of Chicago, 5640 S. Ellis Ave., Chicago, IL \\ 60637, USA \\ $\left({ }^{*}\right.$ Author for correspondence, Email: frisch@uchicago.edu)
}

\begin{abstract}
.
The properties of interstellar matter at the Sun are regulated by our location with respect to a void in the local matter distribution, known as the Local Bubble. The Local Bubble (LB) is bounded by associations of massive stars and fossil supernovae that have disrupted dense interstellar matter (ISM), driving low density intermediate velocity ISM into the void. The Sun appears to be located in one of these flows of low density material. This nearby interstellar matter, dubbed the Local Fluff, has a bulk velocity of $\sim 19 \mathrm{~km} \mathrm{~s}^{-1}$ in the local standard of rest. The flow is coming from the direction of the gas and dust ring formed where the Loop I supernova remnant merges into the LB. Optical polarization data suggest the local interstellar magnetic field lines are draped over the heliosphere. A longstanding discrepancy between the high thermal pressure of plasma filling the LB and low thermal pressures in the embedded Local Fluff cloudlets is partially mitigated when the ram pressure component parallel to the cloudlet flow direction is included.
\end{abstract}

Keywords: ISM: general, ISM: abundances

\section{Introduction}

The existence of an area clear of interstellar material around the Sun, now known as the Local Bubble, was discovered as an underdense region in measurements of starlight reddening (Fitzgerald, 1968). This underdense region is traced by color excess measurements showing $E(B-V)<0.01 \mathrm{mag},{ }^{1}$ and extends beyond 100 $\mathrm{pc}$ in the galactic longitude interval $\ell=180^{\circ}-270^{\circ}$. In the plane of Gould's Belt, the Local Bubble boundaries ("walls") are defined by interstellar material (ISM) associated with star forming regions. At high galactic latitudes the Local Bubble boundaries are defined by interstellar gas and dust displaced by stellar evolution, particularly supernova in the Scorpius-Centaurus Association. Supernovae exploding into pre-existing cavities created by massive star winds displace ISM and the interstellar magnetic field into giant magnetized bubbles hundreds of parsecs in extent. The location of the Sun within such a void regulates the interstellar radiation field at the Sun, and the composition and properties of the ISM surrounding the heliosphere.

The Local Interstellar Cloud (LIC), defined by the velocity of interstellar $\mathrm{He}^{\circ}$ inside of the heliosphere, is one cloudlet in a low density ISM flow known as the Local Fluff. The Local Fluff has an upwind direction towards Loop I and the

\footnotetext{
${ }^{1} E(B-V)=A_{\mathrm{B}}-A_{\mathrm{V}}$, where $A_{\mathrm{B}, \mathrm{V}}$ is the attenuation in units of magnitude in the blue (B) and visible (V) bands, respectively.
}

(c) 2018 Springer Science + Business Media. Printed in the USA. 
Scorpius-Centaurus Association (SCA). This flow, with a best-fit local standard of rest (LSR) velocity of $\approx 19.4 \mathrm{~km} \mathrm{~s}^{-1}$, appears to be a break-away fragment of the Loop I superbubble shell surrounding the SCA, which has expanded into the low density interior of the Local Bubble (§2.1, Frisch, 1981; Frisch, 1995; Breitschwerdt et al., 2000).

This paper is in honor of Prof. Johannes Geiss, founder the International Space Sciences Institute (ISSI). Many of the contemporary space topics discussed at ISSI meetings, such as the heliosphere, the Local Interstellar Cloud, cosmic ray acceleration and propagation, and the composition of matter, are influenced by the solar location inside of the Local Bubble.

\section{Origin and Boundaries of the Local Bubble}

\subsection{ORIGIN}

The Local Bubble void has been created by star formation processes that have occurred during the past 25-60 Myrs in the corotating region of the Milky Way Galaxy near the solar location of today. About 25-60 Myrs ago a blast wave evacuated a low density region at the present location of the Sun, and compressed surrounding molecular clouds to initiate the formation sequence of the massive $\mathrm{OB}$ stars now attributed to Gould's Belt. Gould's Belt denotes the system of kinematically related massive OB stars within $\sim 500 \mathrm{pc}$ of the Sun, which form a localized plane tilted by $\sim 18^{\circ}$ with respect to the galactic plane. The center of Gould's Belt is $104 \mathrm{pc}$ from the Sun towards $\ell=180^{\circ}$, and with an ascending node longitude of $296^{\circ}$ (Grenier, 2004). The Sun is moving away from the center of Gould's Belt, and is closest to the Scorpius-Centaurus rim. Overlapping superbubbles shape the Local Bubble void (Frisch, 1995; Heiles, 1998; Maiz-Appellaniz, 2001).

Since the formation of Gould's Belt, the Sun has traveled hundreds of parsecs through the LSR, and the LSR has completed $\sim 10-25 \%$ of its orbit around the galactic center. Molecular clouds disrupted by the initial blast wave now rim Gould's Belt. Epochs of star formation in the Scorpius-Centaurus Association during the past 1-15 Myrs further evacuated the Local Bubble void, and displaced ISM from the SCA into giant nested $\mathrm{H}^{\circ}$ shells (de Geus, 1992). One of these shells, Loop I (the North Polar Spur), was formed by a recent supernova ( $<1$ Myrs ago) and is an intense source of polarized synchrotron and soft X-ray (SXR) emission. A ring-like shadow, caused by foreground ISM, is seen in the Loop I SXR emission. The origin of this ring has been suggested to be the result of Loop I merging with the separate Local Bubble (e.g. Egger and Aschenbach, 1995). The LSR upwind direction of the Local Fluff is at the center of this ring, and polarization data show that the ring is a magnetic loop (Fig. 1). ${ }^{2}$

\footnotetext{
${ }^{2}$ In accordance with general practice, here I use the Standard solar apex motion (velocity 19.7 $\mathrm{km} \mathrm{s}^{-1}$, towards $\ell=57^{\circ}$ and $b=22^{\circ}$ ) to correct heliocentric velocities to the LSR. This gives a Local
} 


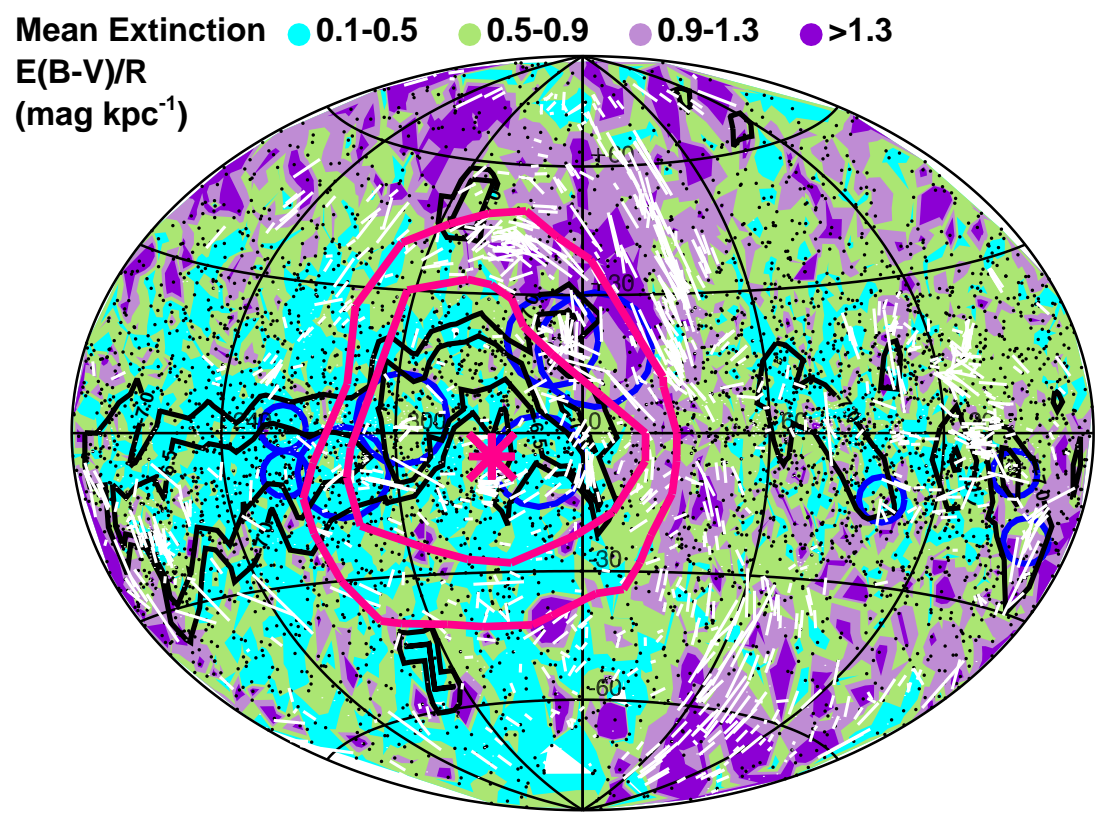

Figure 1. Mean extinction, $E(B-V) / R$, for stars with $R<0.5$, where $R$ is the distance in kpc. Black contours show the integrated stellar radiation at 1565 A measured by the TD- 1 satellite (Gondhalekar et al., 1980). Contours indicate flux levels of $10^{-7}$ and $10^{-6.5} \mathrm{ergs} \mathrm{cm}^{-2} \mathrm{~s}^{-1} \mathrm{~A}^{-1}$ sterad $^{-1}$. Polarization data for stars within 500 pc of the Sun are plotted as white bars (Heiles 2000). Pink contours show the ring that may be formed by the Loop I supernova remnant interaction with the Local Bubble (Egger and Aschenbach, 1995). The pink asterisk indicates the LSR upwind direction of the Local Fluff (see text). Blue circles show stellar OB associations within $500 \mathrm{pc}$ of the Sun. An Aitoff projection is used, with the galactic center at the center of the plot, and $\ell$ increasing to the right. Note that the ISM density is very low out to $500 \mathrm{pc}$ in the interval $\ell=180^{\circ} \rightarrow 300^{\circ}$, particularly towards low galactic latitudes. This plot is based on photometric data in the Hipparcos catalog. The stars (small black dots) are preselected for photometric quality, and color excess values are smoothed for stars with overlapping distance uncertainties and within $13^{\circ}$ of each other on the sky. The average distances of stars sampling latitudes in the intervals $|b|<\left[30^{\circ}, 30^{\circ}-60^{\circ},>60^{\circ}\right]$ are $[210,164,138] \mathrm{pc}$, so that the ISM in high latitude sightlines tends to be closer. Uncertainties on $E(B-V)$ for the plotted stars are typically $<0.02$ mag because stars are also required to have photometric distances that are consistent with astrometric distances to within $\sim 30 \%$. Intrinsic stellar colors are from Cox (2000).

Fluff LSR upwind direction towards $\ell, b=331^{\circ},-5^{\circ}$, and LSR velocity $-19.4 \mathrm{~km} \mathrm{~s}^{-1}$. In Frisch et al. (2002), we instead used an LSR conversion based on Hipparcos data, and found an LSR upwind direction $\ell=2.3^{\circ}, b=-5.2^{\circ}$ (velocity $-17 \mathrm{~km} \mathrm{~s}^{-1}$ ), which is directed towards the ISM shadow itself. This difference in the two possible Local Fluff upwind directions reveals an obvious flaw in comparing a dynamically defined direction with a statically defined diffuse object such as the more distant SXRB shadow. 


\subsection{BOUNDARIES}

The locations of the Local Bubble boundaries have been diagnosed with a range of different ISM markers, including color excess (Lucke, 1978), ultraviolet observations of interstellar $\mathrm{H}^{\circ}$ lines in hot stars (York \& Frisch, 1983, Paresce, 1984), radio $\mathrm{H}^{\circ}$ 21-cm and optical $\mathrm{Na}^{\circ}$ data (Vergely et al., 2001), extreme ultraviolet (EUV, $\lambda<912 \mathrm{~A}$ ) emission of white dwarf and M-stars (Warwick, 1993), measurements of polarization of starlight (Leroy, 1999), and the trace ionization species $\mathrm{Na}^{\circ}$ (Sfeir et al., 1999, Lallement et al. 2003). These studies differ in sampling densities and spatial smoothing methods. Each marker is an imprecise tracer of the total ISM mass density, since the ISM is highly inhomogeneous over the scale lengths of the Local Bubble and small scale structure is poorly understood. I focus here on the reddening data.

An accessible measure of starlight reddening is color excess, $E(B-V)$, which measures the differential extinction of starlight in the blue versus visual bands, and is sensitive to interstellar dust grains (ISDG) of radii $a \sim 0.20 \mu \mathrm{m}$. Interstellar gas and dust are generally well mixed, so that the threshold reddening for the Local Bubble walls found from $E(B-V)$ data is consistent with the locations found from gas markers. The exception is that dust is found in both neutral and ionized regions, while the commonly available gas markers $\left(\mathrm{H}^{\circ}, \mathrm{Na}^{\circ}\right)$ are weighted towards neutral regions.

Grains and gas are well mixed partly because both populations couple to the interstellar magnetic field $\left(B_{\mathrm{IS}}\right)$ in cold and warm clouds. In cold clouds with density $n(\mathrm{H})=100 \mathrm{~cm}^{-3}$ and temperature $T \sim 100 \mathrm{~K}$, the $a \sim 0.2 \mu \mathrm{m}$ dust grain with density $2 \mathrm{~g} \mathrm{~cm}^{-3}$ will sweep up its own mass in gas in $\sim 0.08$ Myrs. If the same grain has charge $\mathrm{Z}=20$, the gyrofrequency for a magnetic field of strength $\mathrm{B}=2.5 \mu \mathrm{G}$ is $\sim 1 / 3300$ yrs. For a warm neutral cloud $\left(n(\mathrm{H}) \sim 0.25 \mathrm{~cm}^{-3}, \mathrm{~T} \sim 6300\right.$ $\mathrm{K})$, the grain accumulates its own mass in $\sim 4$ Myrs. In both cloud types, grains couple to $B_{\mathrm{IS}}$. Gas also couples to $B_{\mathrm{IS}}$, since elastic collisions couple neutrals and ions over time-scales of years, and minimum ionization levels of $\sim 10^{-4}$ bind gas to $B_{\mathrm{IS}}$ (Spitzer, 1978).

In Fig. 1, the reddening per unit distance, $E(B-V) / R$, where $R$ is the star distance in kpc, is shown on an Aitoff projection for O, B, and A stars within 500 pc. $E(B-V)$ values are based on Hipparcos photometric data (Perryman et al., 1997). The lowest mean ISM densities in the galactic plane are between longitudes of $210^{\circ}$ and $360^{\circ}$ are evident. Star groups (blue circles) in the low density sightlines include Sco OB2, Vela OB2, and Trumpler 10 (de Zeeuw et al., 1999). The lowest mean densities in this data set, outside of the Local Fluff, correspond to 0.006 atoms $\mathrm{cm}^{-3}$. At the galactic poles, $|b|>75^{\circ}$, the edges of the Local Bubble, where $E(B-V)>0.05$ mag (or approximately $\log N(\mathrm{H})>20.50 \mathrm{~cm}^{-2}$ ), ${ }^{3}$ are at $80-95$ pc towards both the north and south poles.

\footnotetext{
${ }^{3}$ The ratio $E(B-V) / N\left(\mathrm{H}^{\circ}\right)$ varies in sightlines with low mean extinctions because of variations in both mean grain sizes and hydrogen ionization.
} 
The LB boundaries in the galactic plane are shown in Fig. 2, for $\sim 2000$ O, B, and A stars within $\sim 200 \mathrm{pc}$ and $45^{\circ}$ of the galactic plane, using a threshold cumulative value of $\log N(\mathrm{H})>20.4 \mathrm{~cm}^{-2}$ corresponding to $E(B-V)>0.04 \mathrm{mag}$ when $N(\mathrm{H}) / E(B-V) \sim 5.8 \times 10^{21} \mathrm{~cm}^{-2} \mathrm{mag}^{-1} \mathrm{~K}$. This gas-to-dust ratio is good to within factors of $\sim 2$ for $E(B-V)>0.1$ mag and $\sim 3$ for $E(B-V)<0.1$ mag (Bohlin et al., 1978). Note the well known deficiency of ISM out to distances beyond $200 \mathrm{pc}$ in the third and parts of the fourth galactic quadrants (Frisch and York, 1983). For cloudy sightlines (high mean $E(B-V)$ values), the fraction of the $\mathrm{H}$ atoms in $\mathrm{H}_{2}\left(f_{\mathrm{H}_{2}}\right)$ rises above $\sim 1 \%$ at $E(B-V) \sim 0.1$ mag. The classic term "intercloud" refers to low column density sightlines with relatively little $\mathrm{H}_{2}$ $\left(f_{\mathrm{H}_{2}}<1 \%\right)$. Molecular clouds of $\mathrm{CO}$ and $\mathrm{H}_{2}$ are also shown, and are plotted as filled red circles (Dame et al., 2001). Well-known molecular clouds at the rim of the Local Bubble include dust in Scorpius $\left(\ell \sim 350^{\circ}, \mathrm{d} \sim 120 \mathrm{pc}\right)$, Taurus $\left(\ell \sim 160^{\circ}\right.$, $\mathrm{d} \sim 120 \mathrm{pc})$, and Chameleon $\left(\ell \sim 305^{\circ}, b \sim-15^{\circ}, \mathrm{d} \sim 165 \mathrm{pc}\right)$.

The mean value of $E(B-V) /\left(N\left(\mathrm{H}^{\circ}\right)+2 N\left(\mathrm{H}_{2}\right)\right)$ varies by $\sim 15 \%$ between sightlines with low and high fractions of $\mathrm{H}_{2}$ (Bohlin et al., 1978), because of variations in the mean grain size and radiation field. The $\lambda \sim 1565$ A radiation field depends on location with respect to the Local Bubble walls ( $\S 4.1)$, and the 912-1108 A radiation field capable of photodissociating $\mathrm{H}_{2}$ should behave in a similar fashion.

\section{Loop I and the Local Magnetic Field}

\subsection{Loop I ANd the High-Latitude Limits of the Local Bubble}

Above the galactic plane in the galactic-center hemisphere, $b>20^{\circ}$, the LB walls are established by neutral gas of the Loop I superbubble. The interval $\ell \sim 270^{\circ} \rightarrow$ $50^{\circ}$ is encircled by high-latitude nested shells of gas and dust. Loop I is $\sim 80^{\circ}$ in radius and centered $120 \mathrm{pc}$ away at $\ell=320^{\circ}, b=5^{\circ}$ for the neutral gas (Berkhuijsen 1971; Heiles, 1998; de Geus, 1992). The central regions of these evacuated shells are deficient in ISM, creating the extension of the Local Bubble towards $\ell \sim 340^{\circ}$ (Fig. 2).

\subsection{MAGNetic Field}

Loop I dominates the magnetic field structure near the Sun, and is a source of intense radio continuum and soft X-ray emission. The Loop I magnetic field, comprised of components parallel $\left(B_{\|}\right)$and perpendicular $\left(B_{\perp}\right)$ to the sightline, is traced by polarized synchrotron emission, starlight polarization caused by magnetically aligned dust grains, Faraday rotation, and Zeeman splitting of the $\mathrm{H}^{\mathrm{O}}$ 21-cm line. Fig. 1 shows the starlight polarization vectors (from Heiles, 2000). Magnetically aligned interstellar dust grains (ISDGs) are birefringent at optical wavelengths, with lower opacities found for the polarization component parallel to 


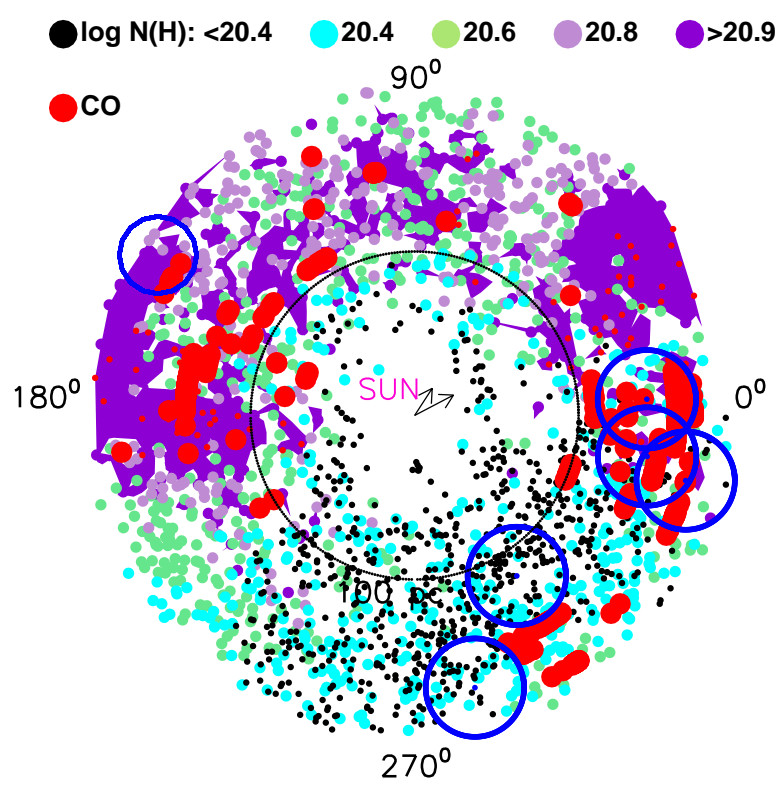

Figure 2. The distribution of ISM surrounding the Local Bubble void, based on stars within $200 \mathrm{pc}$ and within $45^{\circ}$ of the galactic plane. Molecular clouds of $\mathrm{CO}$ and $\mathrm{H}_{2}$ are plotted as red symbols (Dame et al. 2001). The colored dots show cumulative hydrogen column densities towards O, B, and A stars based on $E(B-V)$ (Fig. 1) and the gas-to-dust ratio measured by the Copernicus satellite for stars with low mean extinctions (see text). The mean gas-to-dust relation overestimates $N(\mathrm{H})$ at low column densities $\left(N(\mathrm{H})<10^{20} \mathrm{~cm}^{-2}\right.$, Bohlin et al. 1978). Sightlines with $N(\mathrm{H})>10^{20.9}$ $\mathrm{cm}^{-2}$ have been plotted with filled purple contours. The arrows show two different values for the direction of the Sun's motion through the LSR, with the longer arrow $\left(v=19.5 \mathrm{~km} \mathrm{~s}^{-1}\right)$ indicating the Standard solar apex motion. Blue circles indicate OB associations within $200 \mathrm{pc}$ of the Sun. The black circle indicates $100 \mathrm{pc}$.

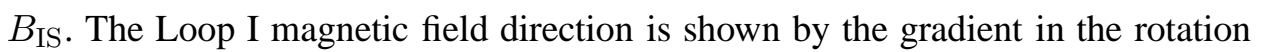
angle of the optical polarization vectors, which follows the interaction ring feature. Comparisons between the optical polarization data (tracing $B_{\|}$) and synchrotron emission (tracing $B_{\perp}$ ), indicate that $B_{\mathrm{IS}}$ is nearly in the plane of the sky in Loop I (Berkhuijsen, 1972, Heiles \& Crutcher, 2005).

The closest measured $B_{\mathrm{IS}}$ strengths are towards Loop I. Heiles et al. (1980) found a volume averaged field strength of $B_{\mathrm{IS}} \sim 4 \mu \mathrm{G}$ in a tangential direction through the shell (extending $\sim 70 \pm 30$ pc towards $\ell=34^{\circ}, b=42^{\circ}$ ). Faraday rotations of extragalactic radio sources indicate that $B_{\|}$is small, with an average value of $B_{\|}=0.9 \pm 0.3 \mu \mathrm{G}$ from rotation measure data (Frick et al., 2001). Magnetic pressure dominates in the neutral shell gas. In the ionized gas producing 
the radio continuum emission, the magnetic, gas, and cosmic ray pressures are all significant. Loop I is a decelerated shock generated by sequential epochs of star formation in SCA (de Geus, 1992)

\subsection{The LiC And the Magnetic Field at the Sun}

The LIC is very low density, $n(\mathrm{H}) \sim 0.25-0.30 \mathrm{~cm}^{-3}$. Magnetic fields in high density ISM show evidence of flux freezing; however $B_{\text {IS }}$ in low density ISM appears uncorrelated with density (Heiles \& Crutcher, 2005). Pulsar dispersion measures indicate that the uniform component of the magnetic field near the Sun is $B_{\mathrm{IS}} \sim 1.4 \mu \mathrm{G}$, with correlation lengths of $\sim 100 \mathrm{pc}$ (Rand \& Kulkarni, 1989). In general, structure functions created from data on radio continuum polarization near $21 \mathrm{~cm}$ show that magneto-ionized structures in interarm sightlines must be very large (e.g. $\sim 100$ pc, Haverkorn et al., 2006). This would indicate that the uniform $B_{\mathrm{IS}}$ component is appropriate for the low density (similar to interarm) region around the Sun.

The physical conditions of the LIC have been modeled by developing a series of radiative transfer models that are constrained by observations of $\mathrm{He}^{\circ}$ and pickup ion and anomalous cosmic ray data inside of the heliosphere, and observations of the LIC towards $\epsilon$ CMa. These models are discussed in detail in Slavin and Frisch (this volume, and 2007, hereafter SF07a,b). The best of these models give $n\left(\mathrm{H}^{\circ}\right)=0.19-0.20 \mathrm{~cm}^{-3}, n(\mathrm{e})=0.07 \pm 0.02 \mathrm{~cm}^{-3}, n\left(\mathrm{He}^{\circ}\right)=0.015 \mathrm{~cm}^{-3}$, for cloud temperatures $\sim 6300 \mathrm{~K}$. If the magnetic and gas pressures are equal in the LIC, then the LIC field strength is $B_{\text {LIC }} \sim 2.8 \mu \mathrm{G}$. This value is also consistent with the interface magnetic field strength of $2.5 \mu \mathrm{G}$, adopted in the best model (model 26). However, it is somewhat above the strength of the uniform component of $B_{\text {IS }}$. Since the ISM flow past the Sun has an origin associated with the breakaway of a parcel of ISM from the Loop I magnetic superbubble (Frisch, 1981), perhaps $B_{\text {IS }}$ at the Sun is stronger and perturbed compared to the uniform field, but at lower pressure than the confined parts of the Loop I bubble.

Very weak interstellar polarization caused by magnetically aligned dust grains has been observed towards stars within $\sim 35 \mathrm{pc}$ (Tinbergen, 1982). The polarization was originally understood to arise in the Local Fluff, since the polarization region coincides with the upwind direction of the flow where column densities are highest. More recently, the polarization properties were found to have a systematic relation to ecliptic geometry. The region of maximum polarization is found to be located at ecliptic longitudes that are offset by $\sim+35^{\circ}$ from the large dust grains flowing into the heliosphere, and from the gas upwind direction (Fig. 3, Frisch et al., 1999). Stars with high polarizations also show consistent polarization angles, and in general polarization is higher for negative ecliptic latitudes. These polarization data are consistent with the interpretation that polarizing grains are trapped in $B_{\text {IS }}$ as it drapes over the heliosphere (Frisch, 2005, 2006). When magnetically prealigned (by $B_{\mathrm{IS}}$ ) grains approach the heliosphere, the gas densities are too low to 
collisionally disrupt the alignment, and polarization should indicate the direction of $B_{\mathrm{IS}}$ at the heliosphere. If the alignment mechanism is sufficiently rapid, the alignment strength and direction will also adjust to the interstellar magnetic field direction as it drapes over the heliosphere. Although this interpretation of the polarization data is not confirmed, it fits the physics of dust grains interacting with the heliosphere. Small charged grains such as those that polarize starlight $(a<0.2 \mu \mathrm{m})$ couple to $B_{\mathrm{IS}}$ and are excluded from the heliosphere, while large grains enter the heliosphere where they are measured by various spacecraft (Krueger, this volume). The characteristics of such polarization may vary with solar cycle phase.

\section{Radiation Environment of the Local Bubble}

\subsection{INHOMOGENEOUS RADIATION FIELD AND LOCAL FLUFF IONIZATION}

The interstellar radiation field (ISRF) is key to understanding the physical properties of the LIC and Local Fluff. The sources of the ISRF at the Sun include plasma emission from the Local Bubble interior and supernova remnants, stellar radiation, including from hot white dwarf stars, and emission from a conductive interface between the local fluff and the hot plasma. The spectrum of this field at the surface of the LIC is shown in Slavin and Frisch (this volume).

The spectrum of the ISRF is inhomogeneous because of the energy-dependent opacity of the ISM. For instance, radiation with $\lambda<912$ A (584 A) determines the ionizations of $\mathrm{H}(\mathrm{He})$. Energetic photons capable of ionizing $\mathrm{H}^{\circ}\left(\mathrm{He}^{\circ}\right)$ require $N\left(\mathrm{H}^{\circ}\right) \sim 17.2(17.7) \mathrm{cm}^{-2}$ to reach an opacity $\tau \sim 1$. The dependence of $\tau_{912 \mathrm{~A}} / \tau_{504 \mathrm{~A}}$ on $N\left(\mathrm{H}^{\circ}\right)$ drives the need for LIC photoionization models to determine the heliosphere boundary conditions. Stars within $\sim 10 \mathrm{pc}$ (e.g. Wood et al. 2005) show local column density variations of $\log N\left(\mathrm{H}^{\mathrm{o}}\right) \sim 17.07-18.22 \mathrm{~cm}^{-2}$ dex (assuming $\log \mathrm{D} / \mathrm{H}=-4.7$ ). This yields a range locally of $\tau_{912 \mathrm{~A}}=0.7-10.5$, and shows that ionization must vary between the individual cloudlets comprising the Local Fluff. This variation is confirmed by $\mathrm{N}^{+}$data, which are excellent tracers of $\mathrm{H}^{+}$through charge-exchange. Stars within $70 \mathrm{pc}$ show $\mathrm{N}^{+} / \mathrm{N}^{\circ} \sim 0.1-2$ (SF07a,b). Our LIC radiative transfer models indicate that in the $\mathrm{LIC} \mathrm{H}^{\mathrm{O}}$ ionization provides $\sim 66 \%$ of the cloud heating, and the LIC is $\sim 20-30 \%$ ionized (SF07a,b).

Another example of the inhomogeneous ISRF is provided by the photon flux at $\lambda \sim 1565 \mathrm{~A}$, which TD-1 satellite data show depends on position in the Local Bubble (Gondhalekar et al., 1980). The ISRF at $1565 \mathrm{~A}$ is dominated by hot stars, B or earlier. Radiation at $\lambda \leq 1620$ A regulates the photoionization rate of interstellar $\mathrm{Mg}^{\circ}$, and is an important parameter for the $\mathrm{Mg}^{+} / \mathrm{Mg}^{\circ}$ diagnostic of the interstellar electron density. The ISDG albedo at $\lambda \sim 1565 \mathrm{~A}$ is $\sim 0.5$. Figure 1 shows the flux of 1565 A photons at the Sun, plotted as black contours. The brightest regions of the sky at $1565 \mathrm{~A}$ are in the third and fourth galactic quadrants, $\ell \sim 180^{\circ}-360^{\circ}$, where the mean extinction in the interior of the Local Bubble is low, $E(B-V) / D<0.4$ mag $\mathrm{kpc}^{-1}$. 
The fact that the 1565 A radiation field is enhanced near the galactic plane for $\ell \sim 180 \rightarrow 360^{\circ}$ is relevant to our understanding of the more energetic photons associated with the soft X-ray background (SXRB). Isolated bright SXRB regions are seen, such as the Orion-Eridanus and Loop I enhancements, however no regional enhancement in the SXRB flux is seen corresponding to the bright $\lambda 1565 \mathrm{~A}$ regions.

\subsection{Diffuse Soft X-RAY BACKGROUnd}

The diffuse soft X-ray background (SXRB) is significant both as an ionizing and heating radiation field, and as a fossil that traces the supernovae that formed the Local Bubble.
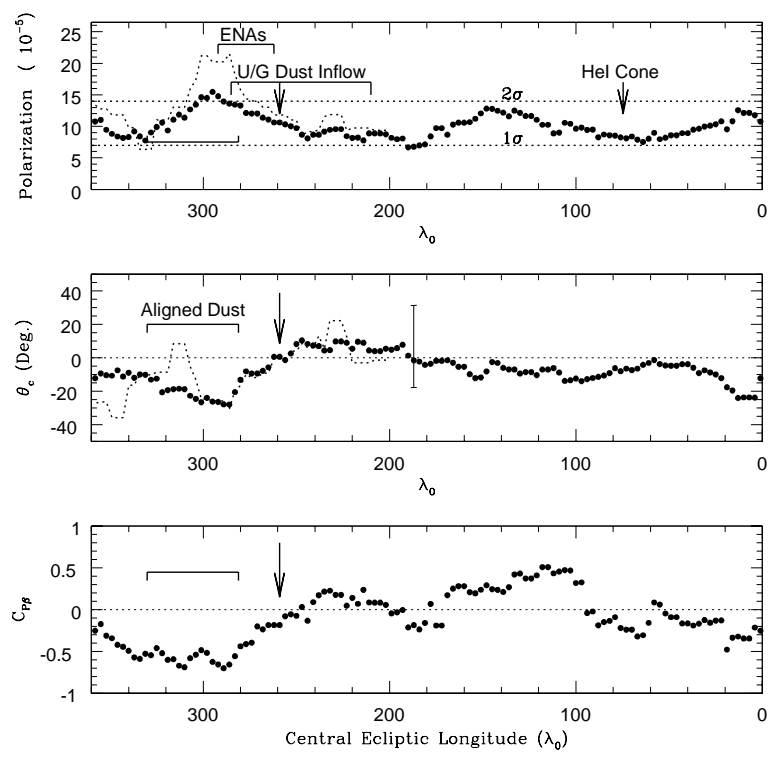

Figure 3. Interstellar polarization towards nearby stars (data from Tinbergen, 1982) compared to the ecliptic position of the star. A systematic enhancement of the polarization strength is found close to the ecliptic at an offset in ecliptic longitude $\lambda$ of $\sim+35^{\circ}$ compared to the inflowing upwind gas and large dust grain directions. Top: The average polarization $P$ for stars with $|\beta|<50^{\circ}$ is plotted as dots, and for stars with $|\beta|<20^{\circ}$ as a dashed line. Data are averaged over $\pm 20^{\circ}$ around the central ecliptic longitude, $\lambda_{\circ}$. The direction of maximum $P$ is shifted by $\sim 25-30^{\circ}$ from the upwind direction of the large interstellar dust grains detected by Ulysses/Galileo (Frisch et al., 1999). The upwind gas and large-grain directions differ by $<5^{\circ}$. Middle: The averaged polarization position angle in celestial coordinates, $\Theta_{\mathrm{c}}$. In the region of maximum polarization, $\lambda \sim 280^{\circ} \rightarrow 310^{\circ}$, the grains show consistent position angles. Bottom: The correlation coefficient between $P$ (top) and $\beta$ is shown as a function of the ecliptic longitude. The strongest polarization is found at negative ecliptic latitudes. For more details see Frisch $(2005,2006)$. 
The spectrum of the soft X-ray background (SXRB) emission determined using broadband sounding rocket observations at energies $\sim 0.08-0.2 \mathrm{keV}$ revealed an excess of count rates at low energies in the galactic plane, compared with the spectrum predicted by an absorbed $T \sim 10^{6} \mathrm{~K}$ plasma. This effect was interpreted as indicating a local X-ray plasma with less than $\sim 7 \times 10^{18} \mathrm{~cm}^{-2}$ of foreground hydrogen (Juda et al., 1991). The complex SXRB spectrum includes this local hot plasma, inferred to fill the LB, along with contributions from absorbed galactic halo emission, extragalactic emission, and absorbed local supernova remnants such as Loop I which dominates the northern hemisphere sky.

Fast-forwarding to the present, the LB contributions to the SXRB have been found from broadband ROSAT data $(0.18-0.3 \mathrm{keV})$ and XMM Newton spectra of dark clouds that help isolate the LB flux from foreground and background fluxes, combined with SXRB models that include foreground contamination from charge-exchange between the solar wind and neutral interstellar or geocoronal gas (e.g. Robertson and Cravens, 2003; Snowden et al., 2004; Bellm and Vaillancourt, 2005; Henley et al. 2007; Lallement, this volume ). The contribution of CEX to the SXRB is significant above $\sim 0.3 \mathrm{keV}$, and is limited by the SXRB flux that does not anticorrelate with $N\left(\mathrm{H}^{\mathrm{o}}\right)$. Models of the contributions of heliospheric charge exchange to the SXRB measured by ROSAT indicate that $\sim 50-65 \%$ of the SXRB in the galactic plane and $\sim 25 \%$ at high galactic latitudes may arise from CEX, depending on the adopted model for CXE fluxes, the solar cycle phase, and ecliptic latitude of the look direction. LB plasma models with depleted abundances predict approximate consistency between the ROSAT and sounding rocket SXRB data. Comparisons between XMM Newton spectra acquired while pointing on and off of a dark cloud in principle allow the removal of foreground heliospheric CEX. For a solar abundance plasma model, based on recent values (Asplund, this volume), the radius of the cavity filled with plasma is $\sim 100 \mathrm{pc}$, with plasma density $n(\mathrm{e})=0.013 \mathrm{~cm}^{-3}$, pressure $2.9 \times 10^{4} \mathrm{~cm}^{-3} \mathrm{~K}$, a cooling time of $17 \mathrm{Myrs}$, and a sound-crossing time of 1.2 Myrs. The LB plasma properties are a topic of ongoing research because the foreground contamination from CEX and contributions to the SXRB spectra contributed by the halo and disk gas are poorly understood.

The LIC ram pressure, $P_{\text {ram }}$, may be important for the pressure equilibrium between the LIC and LB plasma. At the Sun, the SF07 LIC models find a total density of $n\left(\mathrm{H}^{\mathrm{o}}+\mathrm{H}^{+}\right) \sim 0.26$ atoms cm $\mathrm{cm}^{-3}$, and $n(\mathrm{e}) \sim 0.07 \mathrm{~cm}^{-3}$, for $T=6300 \mathrm{~K}$, giving a static LIC pressure of $\sim 2200 \mathrm{~cm}^{-3} \mathrm{~K}$ (including He). For a relative LICLB velocity of $\sim 20.7 \mathrm{~km} \mathrm{~s}^{-1}, P_{\text {ram }} \sim 20,000 \mathrm{~cm}^{-3} \mathrm{~K}$, so that $P_{\text {ram }}$ helps offset the high LB thermal pressure in one dimension. However, for the LIC ram pressure to remove the longstanding mystery about the pressure equilibrium between the LIC and LB plasma, additional pressure contributions (e.g. from magnetic and cosmic ray) are required for directions perpendicular to the relative LIC-hot gas velocity vector. 


\section{The LIC and the ISM Flow Past Sun}

\subsection{Kinematics of Local FlufF}

The LIC is part of an localized ISM flow that has been denoted the Local Fluff, or Cluster of Local Interstellar Clouds (CLIC). The best fitting heliocentric flow vector for ISM within $\sim 30 \mathrm{pc}$ is $-28.1 \pm 4.6 \mathrm{~km} \mathrm{~s}^{-1}$, from the direction $\ell=12.4^{\circ}$, $b=11.6^{\circ}$ (Frisch et al. 2002). In the LSR, the upwind direction is towards the center of Loop I (footnote 2, §2.1). The flow is decelerating; in the rest frame of the flow velocity, the fastest components in the upwind and downwind directions are blueshifted by over $10 \mathrm{~km} \mathrm{~s}^{-1}$. Individual cloudlets contribute to the flow, including the LIC, the "G-cloud" within $1.3 \mathrm{pc}$ in the upwind direction, and the Apex cloud within $5 \mathrm{pc}$ in the upwind direction and extending towards $\ell \sim 30^{\circ}$. These cloudlets have the same upwind directions to within $\sim \pm 10^{\circ}$, indicating a common origin for the cloudlets comprising the Local Fluff. Alternate interpretations using Local Fluff kinematics and temperatures have parsed the flow into $\sim 15$ spatially distorted components (Linsky, this volume). However, velocity components towards stars in the sidewind direction can not clearly distinguish between individual clouds because of velocity blending.

\subsection{INTERSTELLAR ABUNDANCES}

The abundance pattern of elements in interstellar gas is characterized by abundances that decrease with increases in the mean gas density $(<n(\mathrm{H})\rangle)$ or elemental condensation temperature $\left(T_{\text {cond }}\right)$. Most depletion studies are based on long sightlines with blended velocity components. Our LIC radiative transfer models derive elemental abundances corrected for ionization, for a single low density cloud in space (SF07a,b). For the best models in SF07b, LIC abundances are O/H=295$437 \mathrm{ppm}$, compared to solar abundances $460 \mathrm{ppm}$ (Asplund, this volume), and $\mathrm{N} / \mathrm{H}=40-66 \mathrm{ppm}$, compared to solar values $61 \mathrm{ppm}$. The LIC S/H ratios are 14$22 \mathrm{ppm}$, compared to solar values $14 \mathrm{ppm}$. If the LIC has a solar composition, as indicated by anomalous cosmic ray data for ${ }^{22} \mathrm{Ne}$ and ${ }^{18} \mathrm{O}$ (Cummings, this volume), then Asplund solar abundances are preferred over earlier values. Carbon is found to be overabundant by a factor of $\sim 2.6$ compared to solar abundances, which helps maintain the LIC cloud temperature near the observed temperature of $6300 \mathrm{~K}$ through $\mathrm{C}$ fine-structure cooling. The $\mathrm{C}$-abundance anomaly appears to be due to the destruction of carbonaceous grains by interstellar shocks. The carbon overabundance is consistent with the deficit of small carbonaceous grains causing the 2200 A bump and far-ultraviolet rise in the ultraviolet extinction curves in some regions.

Acknowledgements: I thank the organizers of the Geiss-fest for a stimulating meeting, and ISSI for recognizing long ago that the interaction of local ISM and the heliosphere is a fascinating topic. NASA grants NNG05GD36G, NNG06GE33G, 
and NAG5-13107 supported this work. The Hipparcos data-parsing tool was developed by Prof. Philip Chi-Wing Fu, under the auspices of NASA grant NAG511999.

\section{References}

Bellm, E. C. \& Vaillancourt, J. E. 2005, ApJ, 622, 959

Bohlin, R. C., Savage, B. D., \& Drake, J. F. 1978, ApJ, 224, 132

Breitschwerdt, D., Freyberg, M. J., \& Egger, R. 2000, A\&A, 361, 303

Dame, T. M., Hartmann, D., \& Thaddeus, P. 2001, ApJ, 547, 792

de Geus, E. J. 1992, A\&A, 262, 258

de Zeeuw, P. T., Hoogerwerf, R., de Bruijne, J. H. J., Brown, A. G. A., \& Blaauw, A. 1999, AJ, 117, 354

Egger, R. J. \& Aschenbach, B. 1995, A\&A, 294, L25

Fitzgerald, M. P. 1968, AJ, 73, 983

Frick, P., Stepanov, R., Shukurov, A., \& Sokoloff, D. 2001, MNRAS, 325, 649

Frisch, P. C. 1981, Nature, 293, 377

Frisch, P. C. 1995, Space Sci. Rev., 72, 499

Frisch, P. C. 2005, ApJ, 632, L143

Frisch, P. C. 2006, ApJ, submitted

Frisch, P. C., Dorschner, J. M., Geiss, J., et al. 1999, ApJ, 525, 492

Frisch, P. C., Grodnicki, L., \& Welty, D. E. 2002, ApJ, 574, 834

Frisch, P. C. \& York, D. G. 1983, ApJ, 271, L59

Gondhalekar, P. M., Phillips, A. P., \& Wilson, R. 1980, A\&A, 85, 272

Grenier, I. A. 2004, ArXiv Astrophysics e-prints

Haverkorn, M., Gaensler, B. M., Brown, J. C., et al. 2006, ApJ, 637, L33

Heiles, C. 1998, ApJ, 498, 689

Heiles, C. 2000, AJ, 119, 923

Heiles, C., Chu, Y. ., Troland, T. H., Reynolds, R. J., \& Yegingil, I. 1980, ApJ, 242, 533

Heiles, C. \& Crutcher, R. 2005, ArXiv Astrophysics e-prints

Henley, D. B., Shelton, R. L., \& Kuntz, K. D. 2007, ArXiv Astrophysics e-prints

Juda, M., Bloch, J. J., Edwards, B. C., et al. 1991, ApJ, 367, 182

Lallement, R., Welsh, B. Y., Vergely, J. L., Crifo, F., \& Sfeir, D. 2003, A\&A, 411, 447

Leroy, J. L. 1999, A\&A, 346, 955

Lucke, P. B. 1978, A\&A, 64, 367

Maíz-Apellániz, J. 2001, ApJ, 560, L83

Paresce, F. 1984, AJ, 89, 1022

Perryman, M. A. C. 1997, A\&A, 323, L49

Rand, R. J. \& Kulkarni, S. R. 1989, ApJ, 343, 760

Robertson, I. P. \& Cravens, T. E. 2003, Journal of Geophysical Research (Space Physics), 108, 6

Sfeir, D. M., Lallement, R., Crifo, F., \& Welsh, B. Y. 1999, A\&A, 346, 785

Slavin, J. D. \& Frisch, P. C. 2007, A\&A, in preparation

Snowden, S. L., Collier, M. R., \& Kuntz, K. D. 2004, ApJ, 610, 1182

Spitzer, L. 1978, Physical Processes in the Interstellar Medium (John Wiley \& Sons, Inc.)

Tinbergen, J. 1982, A\&A, 105, 53

Vergely, J.-L., Freire Ferrero, R., Siebert, A., \& Valette, B. 2001, A\&A, 366, 1016

Warwick, R. S., Barber, C. R., Hodgkin, S. T., \& Pye, J. P. 1993, MNRAS, 262, 289

Wood, B. E., Redfield, S., Linsky, J. L., Müller, H.-R., \& Zank, G. P. 2005, ApJS, 159, 118 